\title{
Energy Transfer Kinetics in Photosynthesis as an Inspiration for Improving Organic Solar Cells
}

\author{
Collins Nganou, ${ }^{\dagger, \nabla}$ Gerhard Lackner, ${ }^{\ddagger \nabla}$ Bezu Teschome, ${ }^{\S}$ M. Jamal Deen,, Noam Adir, ${ }^{\perp}$ David Pouhe, ${ }^{\#}$ \\ Doru C. Lupascu, ${ }^{\ddagger}$ and Martin Mkandawire* ${ }^{* \dagger}$ \\ ${ }^{\dagger}$ Verschuren Centre for Sustainability in Energy and the Environment, Cape Breton University, 1250 Grand Lake Road, Sydney, Nova \\ Scotia B1P 6L2, Canada \\ ${ }^{\ddagger}$ Institute for Materials Science, University of Duisburg-Essen and Centre for Nanointegration Duisburg-Essen (CeNIDE), Essen \\ 45141, Germany \\ ${ }^{\S}$ Institute of Ion Beam Physics and Materials Research, Helmholtz-Zentrum Dresden-Rossendorf, 01328 Dresden, Germany \\ "Electrical and Computer Engineering, McMaster University, 1280 Main Street, West Hamilton, Ontario L8S 4K1, Canada \\ ${ }^{\perp}$ Schulich Faculty of Chemistry, Technion-Israel Institute of Technology, Haifa, 32000 Israel \\ ${ }^{\#}$ Reutlingen University of Applied Sciences, Alteburgstrase 150, 72762 Reutlingen, Germany
}

Supporting Information

\begin{abstract}
Clues to designing highly efficient organic solar cells may lie in understanding the architecture of light-harvesting systems and exciton energy transfer (EET) processes in very efficient photosynthetic organisms. Here, we compare the kinetics of excitation energy tunnelling from the intact phycobilisome (PBS) light-harvesting antenna system to the reaction center in photosystem II in intact cells of the cyanobacterium Acaryochloris marina with the charge transfer after conversion of photons into photocurrent in vertically aligned carbon nanotube (va$\mathrm{CNT}$ ) organic solar cells with poly(3-hexyl)thiophene (P3HT) as the pigment. We find that the kinetics in electron hole creation following excitation at $600 \mathrm{~nm}$ in both PBS and va-CNT solar cells to be 450 and $500 \mathrm{fs,} \mathrm{respectively.} \mathrm{The} \mathrm{EET} \mathrm{process} \mathrm{has}$ a 3 and 14 ps pathway in the PBS, while in va-CNT solar cell devices, the charge trapping in the CNT takes 11 and 258 ps. We show that the main hindrance to efficiency of va-CNT organic solar cells is the slow migration of the charges after exciton formation.
\end{abstract}

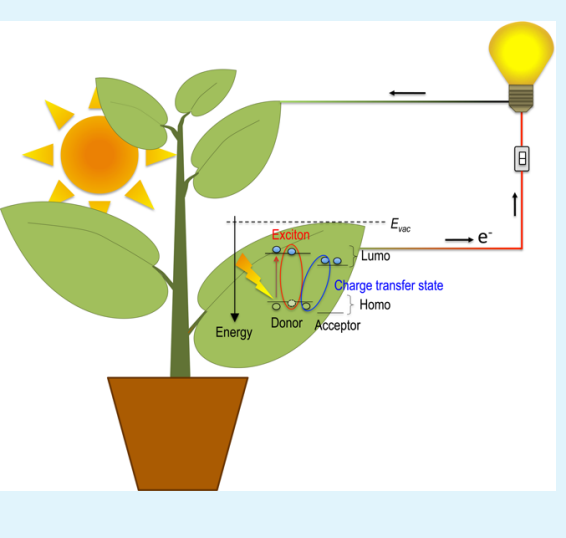

KEYWORDS: chromophore, exciton, polarons, photosynthesis, Acaryochloris marina, photovoltaic, carbon nanotubes, solar energy conversion

\section{INTRODUCTION}

Much of the growing demand for nonpolluting renewable energy sources has focused on the collection and conversion of solar energy (SEC) into electrical current. Inorganic photovoltaic (IPV) cells are one of the strategies for SEC. New materials and fabrication techniques continue to lower the price of manufacture of IPV SEC systems; however, their use continues to be limited. Arguably, IPV adoption has yet to reach a tipping point due to challenges of continued massmarket unaffordability. ${ }^{1,2}$ The prohibitive cost of IPV cells is, intra alia, due to their material and production costs. Thus, alternatives to the expensive inorganic material are required. Researchers have undertaken efforts to develop organic photovoltaics (OPV) as an alternative to IPV. The OPV use relatively inexpensive organic molecules and polymers to absorb solar radiation and convert it to electricity. ${ }^{1-4}$ Further, there are several advantages of OPV, including (a) fabrication and application benefits, low production cost, flexibility, capability of being rolled, printability, and light weight; ${ }^{5,6}$ (b) a wide range of organic-molecule properties, such as high fluorescence efficiency, large polarizability, flexible mechanical properties, ease of processing, and structural diversity; ${ }^{7}$ and (c) the tunable nature of organic-molecule electronic structures, via chemical synthesis. ${ }^{8}$ Despite these advantages, OPV cells still lag far behind IPV cells in efficiency. Currently, OPV cells are about $11.5 \%$ efficient, while in comparison IPV cells are up to $43 \%$ efficient for tandem cells. ${ }^{3}$ The relatively low efficiency of OVP has been attributed to their utilization of a narrow range of the visible spectrum and the excitonic nature of the charge carriers. ${ }^{9}$

The process of converting photoenergy into electrical energy by OVP can be compared to the conversion of solar energy into chemical energy in photosynthesis. They both harvest sunlight using organic pigments and use specific ranges of solar

Received: March 21, 2017

Accepted: May 12, 2017

Published: May 12, 2017 
(a)

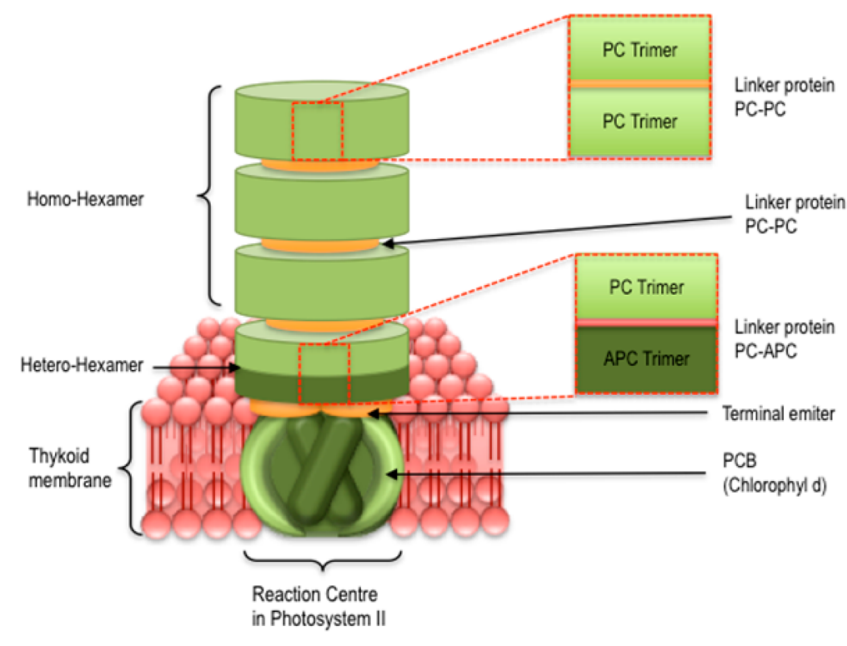

(b)

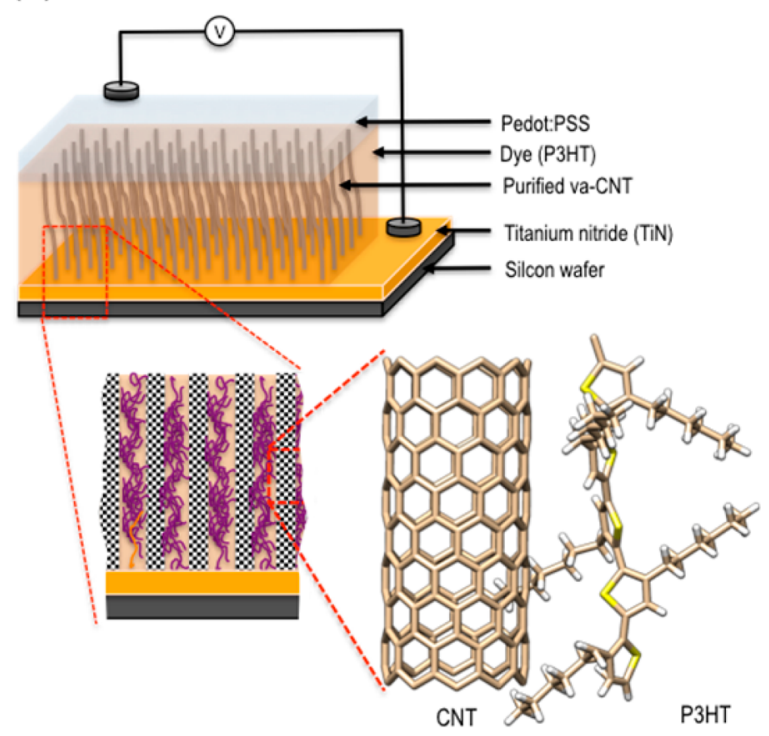

Figure 1. (a) The schematic structural architecture of the PBS of A. marina and (b) architectural assembly of a va-CNT organic solar cell device.

irradiation. However, photosynthesis utilizes a narrower range of solar irradiation than most OVP. ${ }^{10,11}$ The adaptation of some photosynthetic aquatic microorganism like some cyanobacterial species to low light intensity environments ${ }^{12-15}$ suggests that even a narrower range of light absorption can lead to a netpositive efficiency of SEC into chemical energy. This observation suggests that poor charge carrier generation upon exciton dissociation may be a limiting factor in organic solar cells.

In view of the above discussion, we studied comparatively the performance of organic solar cells and the exciton energy transfer (EET) mechanism in cyanobacteria, an efficient photosynthetic aquatic microorganism. Cyanobacteria are prokaryotes that utilize phycobilisomes (PBS), found associated with the thylakoid membrane, as the primary light-harvesting antenna system. The PBS absorbs energy from the sun and then funnels it to the photosynthetic reaction center of photosystem II (PSII), ${ }^{16}$ where the transferred energy drives charge separation and electron transfer processes. The PBS has an efficiency of close to $95 \%$ in light harvesting, which is attributed to its architecture. ${ }^{17-20}$ Therefore, the aim of our comparative study was to understand and compare stages in the process and mechanism of charge carrier generation and transport upon exciton dissociation between the natural and artificial systems. The goal was to identify the sources of inefficiency in solar energy conversion in organic solar cells and use bioinspired designs through mimicking the PBS to improve efficiency.

For comparison, a PBS of Acaryochloris marina strain MBIC11017 and organic solar cell devices assembled using vertically aligned single-walled carbon nanotubes (va-CNT) with poly(3-hexyl)thiophene (P3HT) as the photoactive pigment were studied. The analogues to $\mathrm{P} 3 \mathrm{HT}$ in the PBS are the phycocyanin (PC) and allophycocyanin (APC) components. The assemblies of the PBS system and the vaCNT organic solar cell are illustrated in Figure 1. In brief, the PBS in A. marina is a rodlike structure composed of stacked hexameric disks. ${ }^{19,21}$ Three of the hexamers contain only phycocyanin (PC), while the fourth is a heterohexamer containing PC and allophycocyanin (APC). ${ }^{19,21}$ Each hexamer (and the heterohexamer) is structurally organized by a connection of two trimers through $C_{3}$ symmetry, where the unpigmented proteins, called linkers, are located, ${ }^{22}$ while the trimers themselves are disk-shaped with $C_{3}$ symmetry. The linker proteins have also been suggested to modify the absorption and emission characteristics of nearby pigments. ${ }^{23}$ The P3HT-CNT system was used because of its reported inefficiency despite the incorporation of va-CNT, which was done to create pigment molecule-CNT heterojunctions with organized pigment arrays and should increase transfer of excited energy from the pigment. ${ }^{24,25}$ However, P3HT-CNT devices have conversion efficiencies in the range of 10$15 \%,{ }^{24,25}$ which is not very different from other organic solar cells.

In this paper, we report the similarities and differences between the two systems vis-à-vis the kinetics of the exciton energy transfer (EET) as supported by the system structure in cyanobacteria and the kinetics of the P3HT-CNT system in va-CNT organic solar cell devices. We specifically report on our initial steps to compare the rate of EET in A. marina and vaCNT organic solar cell devices from exciton generation in the pigment to transfer of the charge using transient absorption spectroscopy. Furthermore, we try to identify where the OVP can benefit from the architectural design of the natural system.

\section{EXPERIMENTAL METHODS}

Description of the Studied Systems. Isolated PBS from $A$. marina strain MBIC11017 and organic solar cell devices assembled using va-CNT with $\mathrm{P} 3 \mathrm{HT}$ as the photoactive pigment were used in the study. The details of the growth of A. marina, the procedure of isolating PBS, and the fabrication and assembly of the organic solar cell devices are provided in the Supporting Information (SI).

Kinetic Measurements. To measure the kinetics of energy excitation transfer, femtosecond pump-probe transient absorption techniques were used. The femtosecond pump-probe measurements of the kinetics in PBS were performed in high ionic strength buffer (the specimen investigated in phosphate buffer), which is required to preserve the intactness of the complex. The kinetics in intact cells was monitored without buffer. Similarly, the kinetic measurements of the CNT organic solar cell were measured using the same transient absorption technique as for the PBS. In order to separate the effects of 
(a) Kinetics

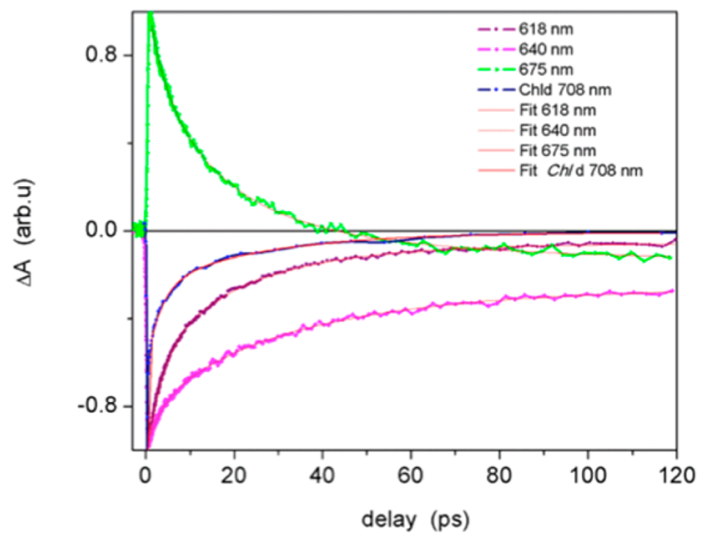

(c) Extracted PBS at room temperature

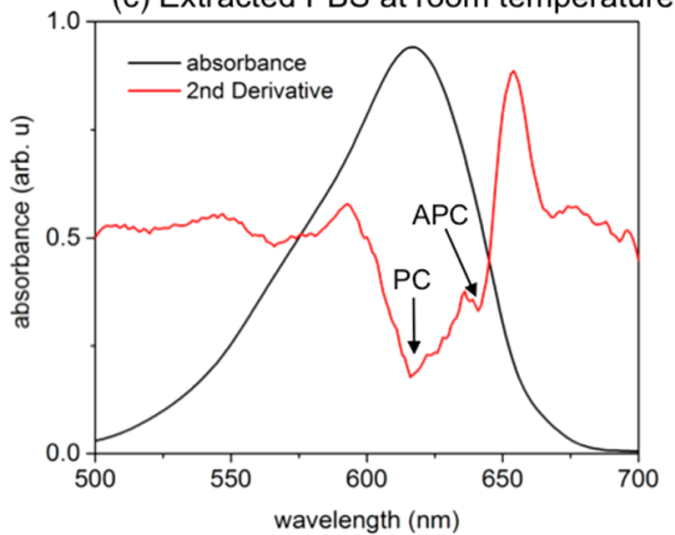

(b) Intact Cell

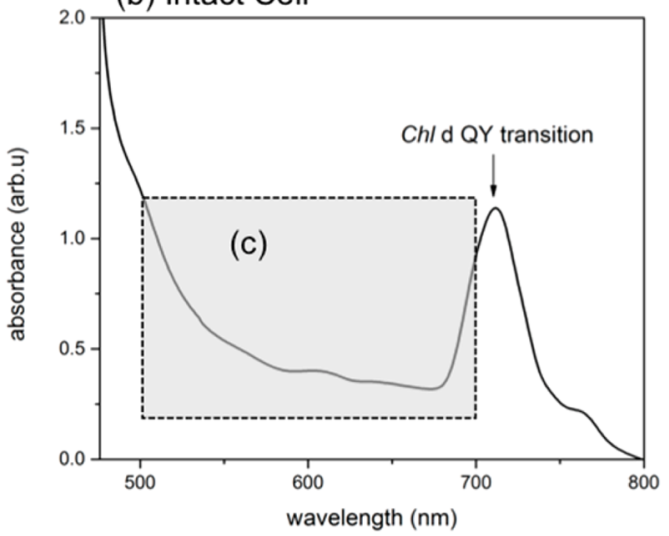

(d) Extracted PBS at $4 \mathrm{~K}$

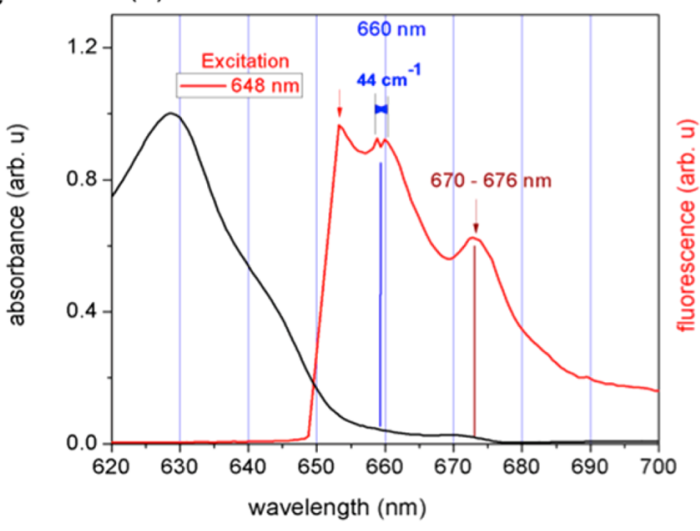

Figure 2. (a) Flash-induced absorption changes at different wavelengths of the antenna rod, especially in PC at $618 \mathrm{~nm}$, APC at $640 \mathrm{~nm}$, the lowenergy emitter at $675 \mathrm{~nm}$, and Chl d bleaching at $708 \mathrm{~nm}$, after excitation at $618 \mathrm{~nm}$. (b) Absorbance of the PBS showing the maximum absorption peak at $618 \mathrm{~nm}$ in PC. The second derivative enhances the observation of the APC at $640 \mathrm{~nm}$. (c) Absorbance of intact cells of A. marina; the QY band of the $\mathrm{Chl} \mathrm{d}$ is observed in the reaction center. (d) Low-temperature absorption and fluorescence emission at $4 \mathrm{~K}$, respectively black and red. The fluorescence spectrum shows high-energy and low-energy exciton state absorption peaks at 660 and $673 \mathrm{~nm}$.

the CNT and the substrate, we manufactured a CNT bucky paper of very high purity functionalized single-chirality semiconducting singlewalled CNT. More than $98 \%$ of the CNT were semiconducting. The optical tests were conducted on these CNT in order to obtain a pure signal and not be perturbed by the large variety of CNT types in a $\mathrm{CNT}$ forest nor by the remaining catalysts or substrate beneath or on top of the forest. To perform transient absorption in bulk, $10 \mathrm{mg}$ of single-walled carbon nanotubes (SWCNT) were diluted in $40 \mathrm{~mL}$ of a mixture of concentrated sulfuric acid $\left(\mathrm{H}_{2} \mathrm{SO}_{4}\right)$ and hydrochloric acid $(\mathrm{HCl})$ at a volume ratio of $3: 1$. This mixture was then sonicated for 2 $\mathrm{h}$ at $70{ }^{\circ} \mathrm{C}$ under reflux, followed by vacuum filtration and neutralization by rinsing with distilled water. The retantate, also known as bucky paper, consisted of the functionalized SWCNT (fSWCNT). Afterward, a piece of that bucky paper $(2 \times 3 \mathrm{~mm})$ was dispersed with the help of a sonotrode (ultrasonic finger) in a reaction vessel in a dispersion medium, 1,2-dichlorobenzene. This procedure was followed by a centrifugation step, and the supernatant was taken, which was the final dispersion. In the f-SWCNT dispersion, the dye was added and stirred for $2 \mathrm{~h}$ at room temperature. One milligram of $\mathrm{f}$ SWCNT with $4 \mathrm{mg}$ of P3HT were eventually dispersed in a $4 \mathrm{~mL}$ solution of 1,2-dichlorobenzene, which resulted in a concentration of $\mathrm{CNT}$ of $0.25 \mathrm{mg} / \mathrm{mL}$. We took $50 \mu \mathrm{L}$ of $\mathrm{f}-\mathrm{SWCNT}+\mathrm{P} 3 \mathrm{HT}$ and mixed it with $300 \mu \mathrm{L}$ of 1,2-dichlorobenzene. A $1 \mathrm{~mm}$ optical path length cuvette was used as sample holder in the pump-probe transient absorption setup. The excitation wavelength was $600 \pm 1 \mathrm{~nm}$ for both PBS and va-CNT organic solar cells.

The setup of pump-probe transient absorption spectroscopy, including the laser system for transient absorption, that was used in the study has been described previously. ${ }^{26}$ Femtosecond laser pulses were produced by a generatively amplified titanium:sapphire laser (SpectraPhysics Spitfire) with a $1 \mathrm{kHz}$ repetition rate. The actinic pump (between 550 and $600 \mathrm{~nm}$ ) was generated via a home-built noncollinear optical parametric amplifier (NOPA). The probe was created by focusing a $800 \mathrm{~nm}$ beam through a $\beta$-barium borate crystal. The obtained $430 \mathrm{~nm}$ beam was further focused onto a calcium fluoride crystal, generating a white light continuum. Pump powers and beam diameters were kept at $\sim 30 \mathrm{~nJ} /$ pulse and $\sim 80 \mu \mathrm{m}$, respectively, with a time resolution of $\sim 100$ fs. A blue color filter (Schott BG38) was used to eliminate the residual $800 \mathrm{~nm}$ light before the detector. The time delay was adjusted by optically delaying the pump pulse, with time steps increasing logarithmically. A chopper blocked every other pump pulse, and a charge-coupled device (CCD) camera (Princeton Instruments, Pixis, 100BR) measured each probe pulse after dispersion by a grating spectrograph (Acton, 150 grates $/ \mathrm{mm}$ ). A custom LabVIEW script controlled monitoring during the translation stage and data acquisition with the detector, and two-dimensional imaging was then used to study the photochemical reaction dynamics.

\section{RESULTS AND DISCUSSION}

\section{Exciton Generation and Trapping in PBS of A. marina.} The process of exciton generation and its kinetics in PBS of $A$. marina has been measured in previous work. ${ }^{27}$ In brief, there are three components of the decay kinetics of 3,14, and $>200$ ps, each of which corresponds to the main absorption peaks at 618,640 , and $673 \mathrm{~nm}$, respectively. Recalling the architecture design of the PBS (see Figure 1), the upper three 
(a)

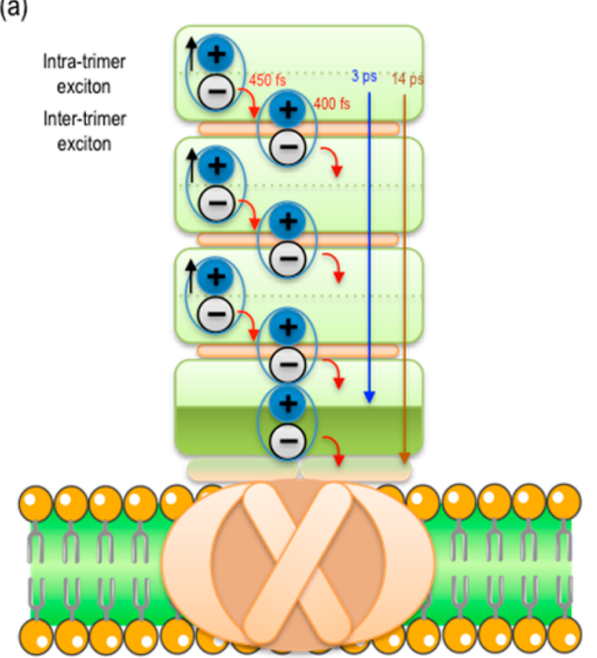

(b)

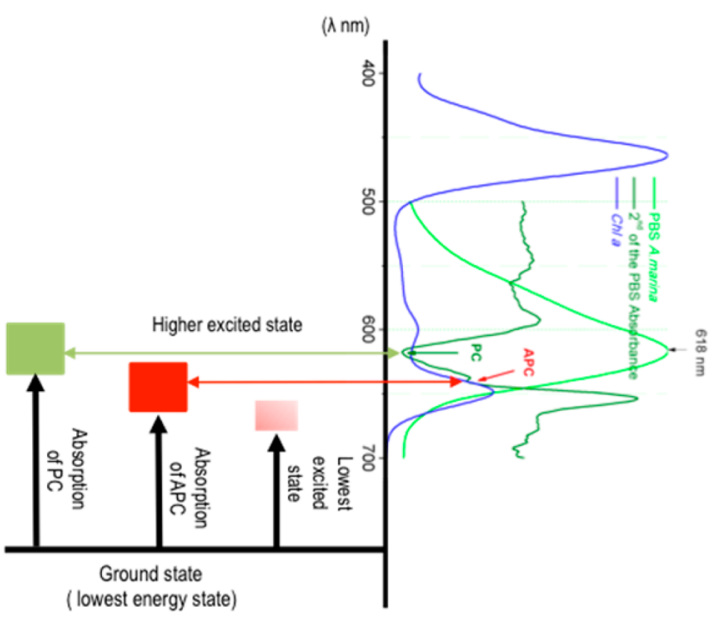

Figure 3. (a) Schematic presentation of PBS architecture summarizing the exciton generation and migration through the trimers. (b) Illustration of the level of downhill excitation energy migration in PBS. After excitation of PC, the absorbed excitation energy is transferred from PC to APC and from APC to the low-energy state in the PBS.

homohexamers contain PC only, and the PC has the maximum electron transition gap of $618 \mathrm{~nm}$ (see Figure $2 \mathrm{~b}$ ). The lower heterohexamer contains both PC and APC. The $618 \mathrm{~nm}$ absorption peak is the excitation of the PC and generation of the exciton, which takes $450 \mathrm{fs}^{27}$ The second derivative of the absorbance shows an absorption peak at $640 \mathrm{~nm}$, which is the maximum transition of APC. This transition takes either 3 or 14 ps via two distinct pathways. ${ }^{27}$ Finally, the charge is trapped from APC to the terminal emitter, which is a localized exciton state, observed at $675 \mathrm{~nm}$. The details of how the localized exciton state (aka terminal emitter) is formed are provided in the Supporting Information.

The PC contains three phycocyanobillin (PCB) cofactors $(\alpha 84, \beta 84$, and $\beta 155)$, which belong to the group of linear tetrapyrrolic pigments. The PCB cofactors absorb between 500 and $675 \mathrm{~nm}$ (Figure 2c). Within this absorption band, there is a distinct transition of specific subsystems. Comparison between measurement at room temperature (Figure 2c) and $4 \mathrm{~K}$ (Figure $2 \mathrm{~d})$ shows that the terminal emitter displays two localized exciton states at 660 and $673 \mathrm{~nm}$ (for an explanation, see the Supporting Information). The trimeric assembly of monomers of PC on which the cofactors are located, as well as their specific positions in the monomer, has been detailed by Kuzminov and co-workers. ${ }^{28}$ The electronic peak absorption changes when the cofactor binds to the protein scaffold via a cysteine residue. $^{22,29}$ The PBS has a distinct absorption peak at $598 \mathrm{~nm}$ in the steady-state absorption at low temperature, which is assigned to the electronic transition of the $\beta 155$ cofactor. ${ }^{30}$ For the exciton to form in PC and APC, the distance between the cofactors should be within $20 \AA^{30,31}$ As a result, exciton formation does not take place in the monomer containing $\beta 155, \alpha 84$, and $\beta 84$ cofactors, since they are more than $20 \AA$ apart. In the trimeric assembly, which brings the rings ( $\mathrm{C}$ and $\mathrm{D}$ ) of cofactors $\alpha 84$ and $\beta 84$ to within $20 \AA$, the rings of cofactors $\alpha 84$ and $\beta 84$ are the main cause of exciton formation in PC and APC. ${ }^{31}$ With the assembly of PBS consisting of three homohexamers of $\mathrm{PC}$ and one heterohexamer of PC trimer and APC trimer, there can be 12 different $\alpha 84(+)-\beta 84(-)$ interactions within photoexcitation. The relaxation kinetics of these excitons in the PBS takes 450 fs. ${ }^{27}$ Furthermore, the rodlike shape of the Am PBS geometry and the fact that there are unpigmented linker proteins positioned along the $C_{3}$ symmetry axis within the hexameric rings suggest that there may be an additional exciton. This exciton is supposed to be formed between $\beta 84(i)$ and $\beta 84(i+$ $1)$, where $i$ denotes the trimer and $i+1$ its adjacent trimer. Nevertheless, the distance between the $\beta 84(i)$ and $\beta 84(i+1)$ in a hexamer without linker is $34 \AA$, which is too large to favor exciton formation. However, the structure of APC from $M$. laminosus deposited in the Protein Data Bank (PDB 1B33) reveals that the nonpigmented linker is in contact with only two $\beta 84$ of the three that are present in a trimer. ${ }^{32}$ Although the linker in structure $1 \mathrm{~B} 33$ is partially homologous to all rod linkers, it is smaller than either the $\mathrm{CpcC}$ rod linkers or the CpcG rod-core linkers. Therefore, it is possible that all the three $\beta 84$ cofactors in a trimer contribute to intertrimer exciton formation. $^{27}$ The three homohexamers in PBS can form nine intertrimer excitons facilitated by the $\mathrm{PC}-\mathrm{PC}$ linker protein. ${ }^{33,34}$ In the heterohexamer unit, the APC trimer shares the same symmetry axis with the PC trimer, and they are linked by the CpcG type of linker protein. The assembly can allow for the formation of an additional exciton between the PC and APC $\beta 84$ cofactors.

The fit of selected kinetic traces between PC, APC, and the terminal emitter to $\mathrm{Chl} \mathrm{d}$ in Figure 2a reveals the 3 and 14 ps kinetic components from PC to the terminal emitter, similar to previous results. ${ }^{27}$ Furthermore, the coupling kinetics between the terminal emitter at $675 \mathrm{~nm}$ and the Chl d at $708 \mathrm{~nm}$ is obtained within two kinetic channels of 3 and 24 ps (Table 1, SI). This suggests that the photoexcitation energy transfer from the PBS to $\mathrm{Chl} d$ in the reaction center of photosystem II happens in less than 70 ps. In every case, we found that exciton relaxation dynamics between $\alpha 84$ and $\beta 84$ as well as $\beta 84(i)$ and $\beta 84(i+1)$ are formed and relaxed before the photoexcitation is transferred to the terminal emitter and, therefore, to the reaction center. For the intertrimer PC exciton, we have found a relaxation dynamic of $400 \mathrm{fs},{ }^{30}$ which means that intertrimer exciton relaxation precedes the intratrimer exciton with relaxation dynamics of $450 \mathrm{fs}$ in the PC of PBS. The structural assembly of PBS is one of the key factors for exciton formation and an efficient exciton flow. 
(a)

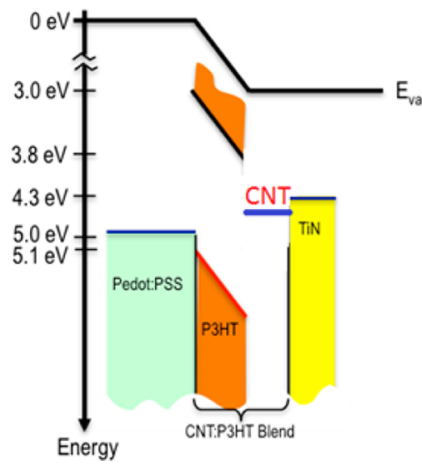

(b)

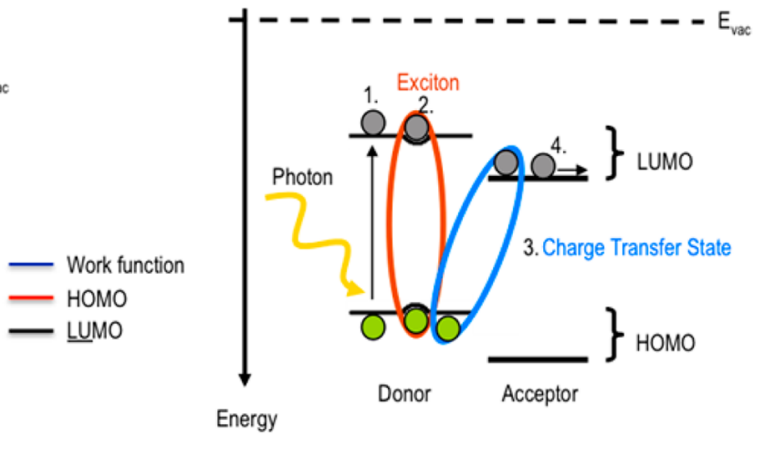

(c)

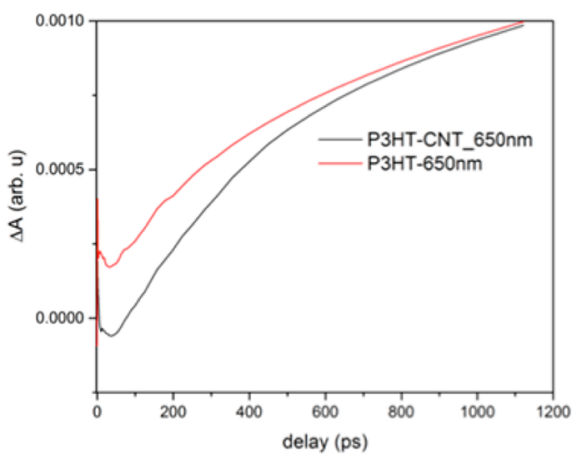

Figure 4. (a) Energy levels of a va-CNT-P3HT organic solar cell showing the band gaps detailing the potential difference with suboptimal energy alignment between CNT and TiN as a consequence of the work function of CNT of $4.7 \mathrm{eV}$. (b) The process of photocurrent conversion in the vaCNT-P3HT solar cell and (c) absorption change of the polaron pair in P3HT and polaron migration from P3HT to CNT. The steps of photocurrent conversion are as follows: (1) incident light is absorbed, causing an electron to shift upward into the LUMO of the donor, while creating the hole in the HOMO; (2) relaxation to an exciton due to the exciton binding energy; (3) the electron hops onto the LUMO of the acceptor due to its higher electron affinity, and the hole remains at the HOMO of the donor, forming the geminate pair together with the electron on the acceptor molecule; and (4) the fully dissociated charge carriers hop as polarons to their respective electrodes (electrons toward the anode and holes toward the cathode).

Exciton Energy Migration from PBS to the Reaction Center. The EET pathways in PBS of A. marina have also been detailed in a previous work. ${ }^{27}$ In Figure $3 a$, we illustrate the formation of excitons, then the photoexcitation energy transfer from the PBS to $\mathrm{Chl} \mathrm{d}$ in the reaction center of photosystem II. The architecture of the PBS allows quick downhill excitation energy migration. When the absorbed light excites the PC component, the absorbed excitation energy is immediately transferred from PC to APC and from APC to the lowest energy state (typically called the terminal emitter). In brief, the PBS absorbs solar radiation between 550 and $660 \mathrm{~nm}$ and forwards it via successively lower energy chromophores. The excitation energy finally migrates from the terminal emitter of $\mathrm{PBS}$ to $\mathrm{Chl}$ in the reaction center. A. marina has $\mathrm{Chl} \mathrm{d}$ instead of $\mathrm{Chl}$ a in the reaction center. The $\mathrm{Chl}$ a absorbs the excited light between 660 and $680 \mathrm{~nm}$, supplied from the terminal emitter of the PBS (Figure 3b). Also, observe the Chl a peak in Figure $3 \mathrm{~b}$. This terminal emitter absorbs the transmitted excited energy from APC and funnels it to the $\mathrm{Chl} \mathrm{d}$, which absorbs at $708 \mathrm{~nm}$ in the reaction center.

Photocurrent Conversion in CNT-P3HT Solar Cells. To determine the mechanism of exciton generation, we analyzed a steady-state absorption spectrum of $\mathrm{P} 3 \mathrm{HT}$, through which we determined the conjugate length and the shoulder of the electronic transition. The $\mathrm{C}=\mathrm{C}$ stretching of $\mathrm{P} 3 \mathrm{HT}$, which can also be observed in Raman scattering at $1450 \mathrm{~cm}^{-1}$ (Figure S5, SI), is responsible for exciton generation. This is observed as a shoulder at around $600 \mathrm{~nm}$ in the steady-state absorption spectrum (SI and Figure 3). This measurement agrees with and supports the theoretical evidence recently reported by Tapping et al. ${ }^{35}$ In bulk form, P3HT falls into the class of polymers capable of adopting different conformations in mixed crystalline and amorphous phases. ${ }^{35,36}$ The amorphous phase exhibits an absorption peak around $450 \mathrm{~nm}$, while the crystalline phase is responsible for the absorption band around 560-640 nm (Figure S4, SI). Thus, the results confirm what was previously reported by Reid et al. that the crystalline phase of P3HT is responsible for high-yield photogeneration. ${ }^{37}$

The process of photocurrent conversion to electrical energy is summarized in Figure 4. Basically, the dye (i.e., P3HT) absorbs the incident light, which brings energy to transfer an electron from the highest occupied molecular orbital (HOMO) to the lowest unoccupied molecular orbital (LUMO), generating an excited state (i.e., an exciton). ${ }^{38}$ Due to the lower dielectric constant $\left(\varepsilon_{\mathrm{r}}\right)$ in organic materials $\left(\varepsilon_{\mathrm{r}} \approx 3-5\right)$ compared to silicon $\left(\varepsilon_{\mathrm{r}}=12\right)$, this excited state forms an exciton. The binding energy of an exciton in organic materials varies in the range from 0.3 to $0.5 \mathrm{eV}$ (Figure $4 \mathrm{~b}$ ). The binding energy of the exciton has to be broken up to separate the two charge carriers: the hole in the HOMO and the electron in the LUMO. ${ }^{39}$ In our device, the generated exciton splits up at the interface of the P3HT and the CNT, and the CNT acts as an electron acceptor. The splitting of the exciton is induced by the $\pi$-electronic valley of CNT, and it was previously reported by Delgado et al. when they investigated graphene deposed on silicon for a graphene-base silicon solar cell. ${ }^{40}$ The splitting of the exciton happens because the $\pi$-electron induces a Coulombic force on the dipole moment of the exciton, which is greater than the exciton-bound Coulombic force. This means that CNT has ionization potential that is greater than that of the excited exciton.

The dissociation of an exciton into separated charge carriers happens in several steps (Figure 5a). The higher electron affinity of the acceptor molecule causes the electron to hop from the LUMO of the donor toward the LUMO of the acceptor at the interface between the donor and acceptor materials. Physically, an electrostatic potential gradient is formed at the donor-acceptor interface, which drives the electron to the LUMO level of the acceptor molecule, while the hole stays at the HOMO of the donor. In this step, the hole and the electron on their respective molecules form a geminate pair, which interacts by Coulombic forces. Thermal activation and electric fields facilitate further separation of the two charge carriers of the geminate pair from each other. When their distance exceeds the Coulomb radius, the two charge carriers are fully dissociated and relax into polarons due to their large effective mass $\left(m_{\mathrm{e}}\right)$. These polarons polarize their neighborhood, hop toward the outer electrodes, and are finally collected at the electrodes. ${ }^{40}$ When the CNT is present in the solar cell device, the kinetics is slow. This slowdown in kinetics is 


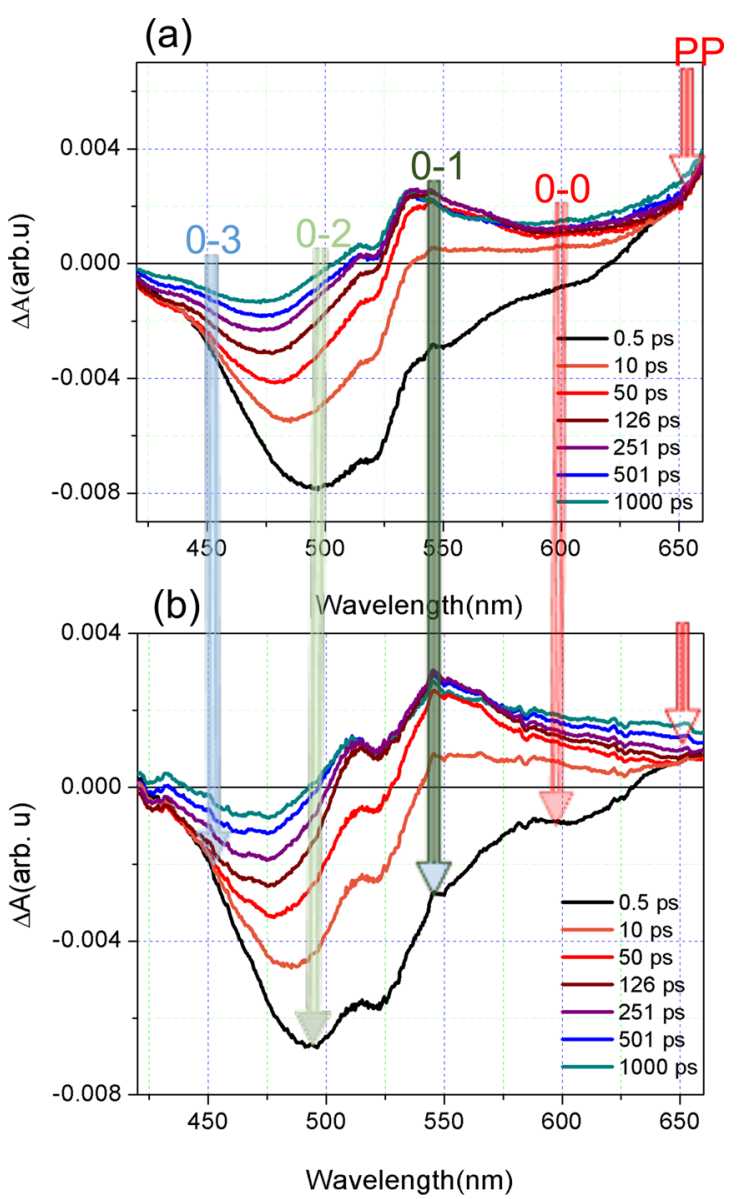

Figure 5. Femtosecond transient absorption (FTA) spectra of (a) $\mathrm{P} 3 \mathrm{HT}$ and (b) $\mathrm{P} 3 \mathrm{HT}+\mathrm{CNT}$ as a function of delay time at $300 \mathrm{~K}$.

attributed to the charge migration from P3HT to CNT (Figure $5 b)$.

Exciton Energy Trapping in the va-CNT Organic Solar

Cells. Femtosecond flash-induced transient absorption spectra of seven selected absorption change traces of the ground state (between 0.5 and 1000 ps) are presented in Figure 5. Measurement of P3HT only and then combined P3HT + va$\mathrm{CNT}$, at $300 \mathrm{~K}$ after excitation at $600 \mathrm{~nm}$, shows similar ground-state photoexcited species between 420 and $550 \mathrm{~nm}$, while the ground-state species above $550 \mathrm{~nm}$ are different. The fluence of the excitation was low enough to avoid formation of single polarons from the hot exciton, ${ }^{41}$ which takes place in less than 0.1 ps. $^{42}$ The polaron was expected to be formed, as described by Ogata et al. ${ }^{43}$ The reference time is defined at the maximum ground-state bleaching at 600,550 , and $500 \mathrm{~nm}$, namely, the $0-0,0-1$, and $0-2$ electronic transitions, respectively. We observed a ground-state bleaching blue-shifted from 496 to $475 \mathrm{~nm}$ in P3HT and P3HT+CNT. The maximum of the ground-state bleaching is the $0-2$ electron transition at $475 \mathrm{~nm}$. Between 580 and $665 \mathrm{~nm}$, a ground-state (GS) bleaching species is formed in $0.5 \mathrm{ps}$ at $600 \mathrm{~nm}$, while an excited-state absorption (ESA) species appears between 623 and $665 \mathrm{~nm}$ in P3HT (Figure 5a). In P3HT+CNT the ESA appears in 0.5 ps at $630 \mathrm{~nm}$ (Figure 5b). After 10 ps, the ESA species appears with the GS bleaching at the 0-0 transition in P3HT, while in P3HT+va-CNT, after 10 ps only the ESA species is present. Possibly, the optical density for the polaron pair (PP) near $650 \mathrm{~nm}$ at the reference time is the maximum peak of ESA. ${ }^{43}$ From this assumption, the conversion of the hot-exciton to PP should appear in a very short time scale (i.e., $<0.5 \mathrm{ps}) .{ }^{43}$ Basco et al. attributes this to an exciton dissociation mechanism in conjugated polymers, which can probably follow two pathways. ${ }^{42,43}$ In the first pathway, it is suggested that the excited vibrational mode decays on the order of the exciton dissociation time scale, which allow more exciton to survive and is equivalent to an effective cooling time of $35 \mathrm{fs}$ of the vibrational heat bath. ${ }^{42}$ The second pathway suggests that all the excitons dissociate first and stay on the same conjugate polymer for some time, which led to local quasi-equilibrium toward recombination to the bound state. ${ }^{42}$ The time scale recombination should be the same as the exciton dissociation. Thus, the vibronic coherence should be lost in time and order of exciton dissociation. Furthermore, use of a low-power pump excitation avoids direct conversion of hot exciton to single polaron. Consequently, a single exciton is formed in the same time scale as PP. This has also been previously report by Reid et al. in their investigation of $\mathrm{P} 3 \mathrm{HT}$ in a film using one femtosecond transient absorption. ${ }^{44}$ They observed the optical density $(\Delta \mathrm{OD})$ of PP at $650 \mathrm{~nm}$ at $t=0$, when the single exciton (S) $\Delta \mathrm{OD}$ appears at $1125 \mathrm{~nm} .{ }^{44}$ Therefore, the conversion of hot exciton to SP or PP appears in a very short time scale, which is also a local quasi-equilibrium mechanism and happens in less than 100 fs. Figure 5a and Figure S1 in the Supporting Information show that the maximum peak does not belong to the reference time zero but instead to the 1000 ps time point.

Recently, the efficiency in charge photogeneration has been correlated to the microstructure of conjugated polymers, like P3HT. ${ }^{37,44,45}$ For instance, semicrystalline P3HT with a chainfolded crystal, which stabilized the amorphous part, was found to be more efficient in photogeneration yield than either the crystalline or amorphous phases of P3HT. In the semicrystalline P3HT, a hole/polaron appears within $0.38 \mathrm{ps}$ after photoexcitation of the electronic transitions at 560 and 604 nm. ${ }^{37}$ The authors claim that exciton relaxation precedes exciton dissociation in P3HT. In a P3HT/PCBM system, Reid et al. suggested that the fastest electron transfer is facilitated by hot exciton dissociation. ${ }^{37}$ In our studies, we obtained waiting times of the spectra in Figure 4, which confirm that the contribution from hot excitons can be neglected in the dynamics observed between 560 and $660 \mathrm{~nm}$. Ogata et al. ${ }^{43}$ recently found a polaron at $650 \mathrm{~nm}$ with the same waiting time as obtained in our current study. ${ }^{43}$ In Figure $5 a$, we observe a broad bleaching of the $0-0$ and $0-1$ electronic transitions. Combining our findings and those of Ogata et al. ${ }^{43}$ suggests the presence of additional photoexcited species, which coexist with the polaron. Previously, Song et al. reported the existence of a species at the $0-0$ electronic transitions and suggested it to be a hole that coexists with a polaron. ${ }^{46}$ This photoexcited hole/ polaron at $600 \mathrm{~nm}$ changes in the presence of CNT (Figure $4 \mathrm{~d})$. Both P3HT and the P3HT-CNT complex have the same ESA, but the bleaching disappears. The disappearance of the bleaching in the presence of CNT at a waiting time above 3 ps (Figure $5 \mathrm{~b}$ ) reveals that the CNT traps the bleaching photoexcited species, which is the hole. A fraction of the PP may also dissociate in the presence of the CNT. This leads to the hole/polaron transfer in the CNT that is mapped by the change in the dynamics of the kinetic with and without CNT in decay-associated ground-state (DASG) bleaching (Figure 6).

Charge Migration. The DASG of the P3HT was obtained with three time constants (9.4 ps, 185 ps, and kinetic $>1 \mathrm{~ns}$ ) 


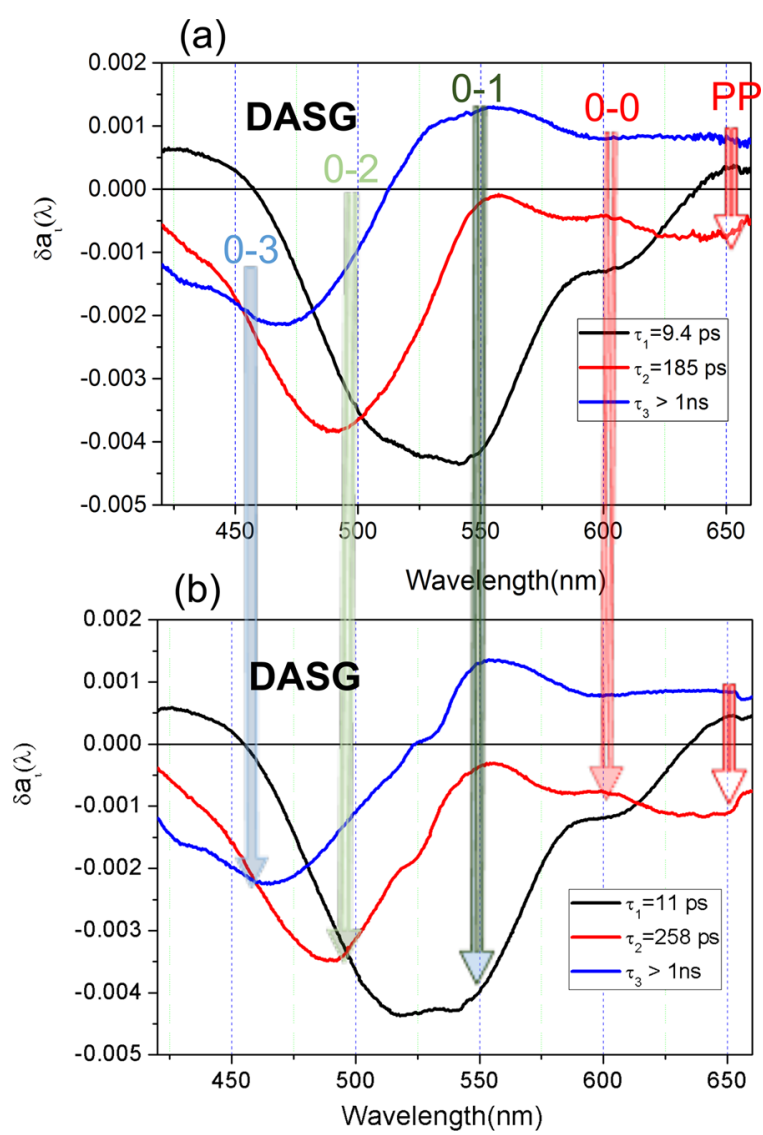

Figure 6. Decay-associated ground-state spectra (DASG) of (a) P3HT and (b) $\mathrm{P} 3 \mathrm{HT}+\mathrm{CNT}$ showing the electronic transition features. The signals at 600 and $650 \mathrm{~nm}$ correspond to hole/polaron pairs (PP).

(Figure 6a). The 9.4 ps component has an ESA peak at $650 \mathrm{~nm}$, which is due to the presence of $\mathrm{PP}$ in its dynamics. The $0-0$ and $0-1$ electronic transitions appeared at 600 and $550 \mathrm{~nm}$, respectively. According to the excitation spectrum in Figure S4 (SI), we activated only the $0-0$ electronic transitions. The bleaching of the $0-1$ electronic transition suggests a fast electronic transfer between the $0-0$ and $0-1$ electronic transitions and subsequent formation of a PP at $650 \mathrm{~nm}$. The electronic energy transfer from the $0-1$ to $0-2$ electronic transition takes place in 9.4 ps. Logically, the $0-1$ electronic bleaching disappears in 185 ps on the dynamics relaxation process. This happens in the presence of a depopulation of $0-1$ electron transition species over 185 ps. Some of the $0-0$ electronic transition species remain, but the peak of excited PP at $650 \mathrm{~nm}$ disappears and a new bleaching transition appears. Therefore, the dissociation of PP and the transfer of electronic energy from $0-2$ to $0-3$ electronic transitions are completed in 185 ps. This result confirms the suggestion by Song et al. that exciton relaxation precedes exciton dissociation in $\mathrm{P}_{3} \mathrm{HT}^{46}$ From the 0-2 transitions, the dissociation likely appears at the interface between the semimetallic and the amorphous sites of P3HT.

In the $\mathrm{P} 3 \mathrm{HT}+\mathrm{CNT}$, the $11 \mathrm{ps}$ component is due to the same mechanism as the 9.4 ps component in P3HT (Figure 6b). The electronic energy transfer in both P3HT and P3HT+CNT is the same except for the time of the transfer process between the electronic transitions. There is a slight increase from 9.4 to 11 ps and a drastic increase of the time of PP dissociation and electronic energy transfer from $0-2$ to $0-3$ electronic transitions. This increase in time of the polaron dissociation from 185 to $258 \mathrm{ps}$ is induced by the presence of two interfaces, namely, (1) the semimetallic and amorphous and (2) the semimetallic and the CNT surface. The second interface (semimetallic and the CNT surface) dominates because of the $\pi$-cationic interaction, ${ }^{47}$ which allows the ring of the CNT to attract the positive electronic site of the PP. At the interface, the quadrupole moment of the CNT ring competes with the Coulombic attraction from the semimetallic-amorphous state. To overcome the competition, the quadrupole moment of the $\mathrm{CNT}$ ring takes on the positive electronic site of the PP. The waiting time in this process is enough to support the increase in time of the dissociation of the PP. The trapping of a hole/ polaron at the CNT was observed with the same kinetics in $\mathrm{P} 3 \mathrm{HT}+\mathrm{CNT}$ dispersed in medium and $\mathrm{P} 3 \mathrm{HT}+\mathrm{va}-\mathrm{CNT}$ because P3HT binds to the surface of CNT through a $\pi$ cationic interaction. But the signal intensity of spectroscopy was higher in $\mathrm{P} 3 \mathrm{HT}+\mathrm{va}-\mathrm{CNT}$ than in medium-dispersed $\mathrm{P} 3 \mathrm{HT}$ $+\mathrm{CNT}$. This can be attributed to the effect of density (lower in dispersing medium than in va-CNT) as well as infiltration of P3HT into the forest of va-CNT (see the SI and Figure S2). For convenience, the CNT were dispersed in P3HT medium to ensure a homogeneous illumination during the measurement of the pump-probe transient spectroscopy. Thus, a solar cell assembled with P3HT well-infiltrated into well-ordered CNT has the possibility of interchain exciton formation due to the infiltrated stacked P3HT between adjacent va-CNT and the possibility of measuring higher exciton formation than in medium-dispersed CNT.

Comparison between Natural and Artificial LightHarvesting Systems. In this comparative study, we have shown that the kinetics of exciton generation, upon excitation at the same wavelength, is almost the same in the pigment PC of the PBS and the pigment P3HT of the organic solar cell. However, we see significant differences in the excitation energy migration from the pigments to the next level. In PBS, the energy is transmitted from the PC/APC to the terminal emitter in two pathways within a time scale of 3 and 14 ps and then finally from the terminal emitter to $\mathrm{Chl} \mathrm{d}$ in the reaction center, also through two pathways within a time scale of 3 and 24 ps. In the va-CNT organic solar cell, the exciton energy is transmitted from P3HT in 258 ps. The ultrafast EET pathways in PBS are enabled by the architecture of the structure between the energy-absorbing antenna and the reaction center. In the organic solar cells, the electron hops from the excited molecule to the adjacent electrode, while in the antenna, the "electrode" surrogate structures are arranged as a pile of rods and the electron hops through the pigment-protein connection.

Generally, charge carrier mobility is quite low in organic solids of amorphous and crystals structure, because the mean free path length of charge carriers is on the order of a lattice constant. This results in hopping transport of free charge carriers in the organic matrix. ${ }^{39}$ va-CNTs are supposed to reduce the mean free path length in the solar cell matrix, functioning as electron recipients because of their ballistic transport properties along their side wall. ${ }^{23,48-51}$ However, examination of Figures 4 and 5 (and also Figures S4 and S5, SI) reveals dispersion spectra of the $\mathrm{CNT}$, representing a mixture of absorption and scattering. According to the literature, absorption in the near-infrared range occurs and the peak at $\sim 950 \mathrm{~nm}$ is based on absorption due to the optical $S_{22}$ transition of the CNT. ${ }^{52}$ Thus, the CNT seems to be responsible for the additional absorption in the near-infrared 
area in the photovoltaic active matrix. The CNTs blended with the polymer $\mathrm{P} 3 \mathrm{HT}$ in the organic solar cell matrix act as acceptor material. ${ }^{53}$ The evidence of CNT influence on the efficiency $(\eta)$ of organic solar cells was assessed by measuring the effectiveness of converting incident photons into collected charge carriers at the electrodes. A spectroscopic analysis using monochromatic light yields the photon-dependent efficiency, also known as the external quantum efficiency (EQE) ${ }^{40}$ The mean $\eta$ values were significantly different between the solar cell devices with and without va-CNT (i.e., 0.03 and 0.63, respectively). In-depth analysis with a scanning electron microscope revealed that the $\eta$ was dependent on interpenetration of P3HT into the forest of the va-CNT (Figure S2, SI). Thus, maintaining the CNT height at a maximum of 120 $\mu \mathrm{m}$ improved the ability of dye to penetrate into the va-CNT forest.

\section{CONCLUSIONS}

In summary, we have shown that it takes almost equal time for exciton formation in photosynthesis pigments in PBS (i.e., PC and APC) of A. marina and P3HT in an organic solar cell device after excitation at $600 \mathrm{~nm}$. However, there is a major kinetic difference between PBS and OVP: the exciton energy tunnelling from its formation to the reaction center is almost 1000 times faster than from P3HT to the electrode, even when vertically aligned CNT are used to reduce the electron-hopping distance. This reveals that the inefficiency of most OVP, including the va-CNT-P3HT system, is mainly due to trapping of the photoexcited energy at the interface of the charge carriers. For this reason, the trapping of the photoexcited bleaching hole by the CNT was observed between 600 and 640 $\mathrm{nm}$. Further, we confirm what was previously reported, that polaron pairs are responsible for the excited-state photogeneration species in bulk $\mathrm{P} 3 \mathrm{HT}$. The polaron pair was observed as a positive band at $650 \mathrm{~nm}$ in both P3HT and $\mathrm{P} 3 \mathrm{HT}+\mathrm{CNT}$. Consequently, the activity of hole dynamics in P3HT after excitation of the $0-0$ transition is attributed to photoexcited species produced by hole/polaron pairs. In view of these preliminary comparisons between the intact PBS system and OVP, the inefficiency of the va-CNT-P3HT system is due to the charge transfer kinetics, which can be improved by using designs inspired by biological processes, materials, and architectures, for instance, the process in the photosynthetic microorganism cyanobacteria.

\section{ASSOCIATED CONTENT}

\section{S Supporting Information}

The Supporting Information is available free of charge on the ACS Publications website at DOI: 10.1021/acsami.7b04028.

Additional methods information (i.e., measuring of EET in A. marina, fabrication of the va-CNT organic solar cell, assembly of the va-CNT OPV, and determination of the porosity of the va-CNT forest) and additional results and discussion (i.e., vertically aligned carbon nanotubes and P3TH infiltration, results of transient resolve spectroscopy analysis of $\mathrm{P} 3 \mathrm{TH}$, ground-state exciton vibration in $\mathrm{P} 3 \mathrm{HT}+\mathrm{CNT}$, energy tunnel kinetics in PBS, and localized exciton state formation (PDF)

\section{AUTHOR INFORMATION}

\section{Corresponding Author}

*E-mail: martin.mkandawire@cbu.ca. Tel +1-902-563-1430. Fax +1-902-563-1360.

ORCID

Martin Mkandawire: 0000-0002-0506-2281

\section{Author Contributions}

${ }^{\nabla}$ C.N. and G.L. contributed equally to this work.

\section{Notes}

The authors declare no competing financial interest.

\section{ACKNOWLEDGMENTS}

All time-resolve experiments were done in the lab of Prof. David MacCamant of the Chemistry Department at Rochester University, Rochester, New York. This work was made possible through the funding to Public Works and Government Services Canada funding (the formerly Enterprise Cape Breton Corp.) to Industrial Research Chair Support, Cape Breton University RP Grant, and different BMBF funding to M.M.; participation of N.A. in the project was supported by US-Israel Bi-National Science Foundation (2014395) and the Israel Science Foundation (1576/12 and 843/16); the contribution from M.J.D. was enabled through NSERC funding; and other support came from TU Dresden, TU Berlin, University of Duisburg-Essen, and Reutlingen University of Applied Sciences.

\section{ABBREVIATIONS USED}

APC, allophycocyanin; Chl, chlorophyll; DASG, decayassociated ground-state spectra; EET, exciton energy transfer; ESA, excited state absorption; EQE, external quantum efficiency; FTA, femtosecond transient absorption; HOMO, highest occupied molecular orbital; IPV, inorganic photovoltaic; LUMO, lowest unoccupied molecular orbital; OPV, organic photovoltaic; P3HT, poly(3-hexyl)thiophene; PBS, phycobilisome; PC, phycocyanin; PCB, phycocyanobillin; PSI, photosystem I; PSII, photosystem II; SEC, solar energy conversion; SWCNT, single-walled carbon nanotubes; vaCNT, vertically aligned carbon nanotube

\section{REFERENCES}

(1) Azarpour, A.; Suhaimi, S.; Zahedi, G.; Bahadori, A. A Review on the Drawbacks of Renewable Energy as a Promising Energy Source of the Future. Arabian J. Sci. Eng. 2013, 38, 317-328.

(2) Breyer, C.; Birkner, C.; Kersten, F.; Gerlach, A.; Goldschmidt, J. C.; Stryi-Hipp, G.; Montoro, D. F.; Riede, M. Research and Development Investments in PV-A Limiting Factor for a Fast PV Diffusion? In 25th European Photovoltaic Solar Energy Conference; WIPRenewable Energies: Munich, Germany, 2009; pp 5385-5408.

(3) Rohr, S. Heliatek Consolidates its Technology Leadership by Establishing a New World Record for Organic Solar Technology with a Cell Efficiency of 12\%; Heliatek GmbH: Dresden, 2013; p 3.

(4) Gregg, B. A.; Hanna, M. C. Comparing Organic to Inorganic Photovoltaic cells: Theory, Experiment, and Simulation. J. Appl. Phys. 2003, 93, 3605-3614.

(5) Ren, S.; Bernardi, M.; Lunt, R. R.; Bulovic, V.; Grossman, J. C.; Gradečak, S. Toward Efficient Carbon Nanotube/P3HT Solar Cells: Active Layer Morphology, Electrical, and Optical Properties. Nano Lett. 2011, 11, 5316-5321.

(6) Krebs, F. C. All Solution Roll-to-roll Processed Polymer Solar Cells Free from Indium-tin-oxide and Vacuum Coating Steps. Org. Electron. 2009, 10, 761-768.

(7) Mitzi, D. B.; Chondroudis, K.; Kagan, C. R. Organic-inorganic Electronics. IBM J. Res. Dev. 2001, 45, 29-45. 
(8) Heath, J. R.; Ratner, M. A. Molecular Electronics. Phys. Today 2003, 56, 43-49.

(9) Wallentin, J.; Anttu, N.; Asoli, D.; Huffman, M.; Åberg, I.; Magnusson, M. H.; Siefer, G.; Fuss-Kailuweit, P.; Dimroth, F.; Witzigmann, B.; Xu, H. Q.; Samuelson, L.; Deppert, K.; Borgström, M. T. InP Nanowire Array Solar Cells Achieving 13.8\% Efficiency by Exceeding the Ray Optics Limit. Science 2013, 339, 1057-1060.

(10) Xu, T.; Yu, L. How to Design Low Bandgap Polymers for Highly Efficient Organic Solar Cells. Mater. Today 2014, 17, 11-15.

(11) Beyer, B.; Leo, K. Efficiency Increase of Organic Solar Cells with Emissive Light-in-Coupling Layers. J. Mater. Chem. C 2015, 3, 1083010836.

(12) Kumazaki, S.; Abiko, K.; Ikegami, I.; Iwaki, M.; Itoh, S. Energy Equilibration and Primary Charge Separation in Chlorophyll d-based Photosystem I Raction Center Isolated from Acaryochloris marina. FEBS Lett. 2002, 530, 153-157.

(13) MacColl, R. Cyanobacterial Phycobilisomes. J. Struct. Biol. 1998, 124, 311-334.

(14) Mackey, K. R. M.; Paytan, A.; Grossman, A. R.; Bailey, S. A Photosynthetic Strategy for Coping in a High-light, Low-nutrient Environment. Limnol. Oceanogr. 2008, 53, 900-913.

(15) Watanabe, T.; Nakamura, A.; Kobayashi, M. Chemical Analytical Approach to the Molecular Machinery of Photosynthesis - Quest for Exotic Chlorophylls in the Reaction Centers. Nippon Kagaku Kaishi 2002, 2, 117-128.

(16) Petrasek, Z.; Schmitt, F.-J.; Theiss, C.; Huyer, J.; Chen, M.; Larkum, A.; Eichler, H. J.; Kemnitz, K.; Eckert, H.-J. Excitation Energy Transfer from Phycobiliprotein to Chlorophyll $\mathrm{d}$ in Intact Cells of Acaryochloris marina Studied by Time- and Wavelength-Resolved Fluorescence Spectroscopy. Photochem. Photobiol. Sci. 2005, 4, 10161022.

(17) Glazer, A. N. Light guides. Directional Energy Transfer in a Photosynthetic Antenna. J. Biol. Chem. 1989, 264, 1-4.

(18) Searle, G. F. W.; Barber, J.; Porter, G.; Tredwell, C. J. Picosecond Time-resolved Energy Transfer in Porphyridium cruentum. Part II. In the Isolated Light Harvesting Complex (Phycobilisomes). Biochim. Biophys. Acta, Bioenerg. 1978, 501, 246-256.

(19) Hu, Q.; Marquardt, J.; Iwasaki, I.; Miyashita, H.; Kurano, N.; Mörschel, E.; Miyachi, S. Molecular Structure, Localization and Function of Biliproteins in the Chlorophyll a/d Containing Oxygenic Photosynthetic Prokaryote Acaryochloris marina. Biochim. Biophys. Acta, Bioenerg. 1999, 1412, 250-261.

(20) Romero, E.; Augulis, R.; Novoderezhkin, V. I.; Ferretti, M.; Thieme, J.; Zigmantas, D.; van Grondelle, R. Quantum Coherence in Photosynthesis for Efficient Solar-energy Conversion. Nat. Phys. 2014, 10, 676-682.

(21) Chen, M.; Floetenmeyer, M.; Bibby, T. S. Supramolecular Organization of Phycobiliproteins in the Chlorophyll d-containing Cyanobacterium Acaryochloris marina. FEBS Lett. 2009, 583, 25352539.

(22) Adir, N. Elucidation of the Molecular structures of Components of the Phycobilisome: Reconstructing a Giant. Photosynth. Res. 2005, $85,15-32$.

(23) Dai, H. Carbon Nanotubes: Synthesis, Integration, and Properties. Acc. Chem. Res. 2002, 35, 1035-1044.

(24) Scharber, M. C.; Sariciftci, N. S. Efficiency of Bulkheterojunction Organic Solar Cells. Prog. Polym. Sci. 2013, 38, 1929-1940.

(25) Deibel, C.; Dyakonov, V. Polymer-fullerene Bulk Heterojunction Solar Cells. Rep. Prog. Phys. 2010, 73, 096401.

(26) Sabatini, R. P.; Eckenhoff, W. T.; Orchard, A.; Liwosz, K. R.; Detty, M. R.; Watson, D. F.; McCamant, D. W.; Eisenberg, R. From Seconds to Femtoseconds: Solar Hydrogen Production and Transient Absorption of Chalcogenorhodamine Dyes. J. Am. Chem. Soc. 2014, 136, 7740-7750.

(27) Nganou, A. C.; David, L.; Adir, N.; Pouhe, D.; Deen, M. J.; Mkandawire, M. Evidence of Additional Excitation Energy Transfer Pathways in the Phycobiliprotein Antenna System of Acaryochloris marina. Photochem. Photobiol. Sci. 2015, 14, 429-438.
(28) Kuzminov, F. I.; Bolychevtseva, Y. V.; Elanskaya, I. V.; Karapetyan, N. V. Effect of APCD and APCF Subunits Depletion on Phycobilisome Fluorescence of the Cyanobacterium Synechocystis PCC 6803. J. Photochem. Photobiol., B 2014, 133, 153-160.

(29) Debreczeny, M. P.; Sauer, K.; Zhou, J.; Bryant, D. A. Comparison of Calculated and Experimentally Resolved Rate Constants for Excitation Energy Transfer in C-Phycocyanin. 2. Trimers. J. Phys. Chem. 1995, 99, 8420-8431.

(30) Nganou, C. Low Absorption State of Phycocyanin from Acaryochloris marina Antenna system: On the Interplay Between Ionic Strength and Excitonic Coupling. J. Chem. Phys. 2013, 139, 045101.

(31) MacColl, R. Allophycocyanin and Energy Transfer. Biochim. Biophys. Acta, Bioenerg. 2004, 1657, 73-81.

(32) Reuter, W.; Wiegand, G.; Huber, R.; Than, M. E. Structural Analysis at 2.2 $\AA$ of Orthorhombic Crystals Presents the Asymmetry of the Allophycocyanin-linker Complex, AP.LC7.8, from Phycobilisomes of Mastigocladus laminosus. Proc. Natl. Acad. Sci. U. S. A. 1999, 96, 1363-1368.

(33) David, L.; Marx, A.; Adir, N. High-resolution Crystal Structure of Trimeric and Rod Phycocyanin. J. Mol. Biol. 2011, 405, 201-213.

(34) David, L.; Prado, M.; Arteni, A. A.; Elmlund, D. A.; Blankenship, R. E.; Adir, N. Structural Studies Show Energy Transfer Within Stabilized Phycobilisomes Independent of the Mode of Rod-core Assembly. Biochim. Biophys. Acta, Bioenerg. 2014, 1837 (3), 385-395.

(35) Tapping, P. C.; Clafton, S. N.; Schwarz, K. N.; Kee, T. W.; Huang, D. M. Molecular-level Details of Morphology-Dependent Exciton Migration in Poly(3-hexythiophene) Nanostructures. J. Phys. Chem. C 2015, 119, 7047-7059.

(36) Dudenko, D.; Kiersnowski, A.; Shu, J.; Pisula, W.; Sebastiani, D.; Spiess, H. W.; Hansen, M. R. A Strategy for Revealing the Packing in Semicrystalline $\pi$-Conjugated Polymers: Crystal Structure of Bulk Poly-3-hexyl-thiophene (Р3HT). Angew. Chem., Int. Ed. 2012, 51, $11068-11072$.

(37) Reid, O. G.; Pensack, R. D.; Song, Y.; Scholes, G. D.; Rumbles, G. Charge Photogeneration in Neat Conjugated Polymers. Chem. Mater. 2014, 26, 561-575.

(38) Deibel, C.; Strobel, T.; Dyakonov, V. Role of the Charge Transfer State in Organic Donor-Acceptor Solar Cells. Adv. Mater. 2010, 22, 4097-4111.

(39) Brabec, C. J.; Heeney, M.; McCulloch, I.; Nelson, J. Influence of Blend Microstructure on Bulk Heterojunction Organic Photovoltaic Performance. Chem. Soc. Rev. 2011, 40, 1185-1199.

(40) Delgado, J. L.; Bouit, P.-A.; Filippone, S.; Herranz, M. a. A.; Martin, N. Organic Photovoltaics: a Chemical Approach. Chem. Commun. 2010, 46, 4853-4865.

(41) Guo, J.; Ohkita, H.; Benten, H.; Ito, S. Near-IR Femtosecond Transient Absorption Spectroscopy of Ultrafast Polaron and Triplet Exciton Formation in Polythiophene Films with Different Regioregularities. J. Am. Chem. Soc. 2009, 131, 16869-16880.

(42) Basko, D. M.; Conwell, E. M. Theory of Hot Exciton Dissociation in Conjugated Polymers. Synth. Met. 2003, 139, 819-821.

(43) Ogata, Y.; Kawaguchi, D.; Tanaka, K. An Effect of Molecular Motion on Carrier Formation in a Poly(3-hexylthiophene) Film. Sci. Rep. 2015, 5, 8436.

(44) Reid, O. G.; Malik, J. A. N.; Latini, G.; Dayal, S.; Kopidakis, N.; Silva, C.; Stingelin, N.; Rumbles, G. The Influence of Solid-state Microstructure on the Origin and Yield of Long-lived Photogenerated Charge in Neat Semiconducting Polymers. J. Polym. Sci., Part B: Polym. Phys. 2012, 50, 27-37.

(45) Halls, J. J. M.; Cornil, J.; dos Santos, D. A.; Silbey, R.; Hwang, D.-H.; Holmes, A. B.; Brédas, J. L.; Friend, R. H. Charge- and EnergyTransfer Processes at Polymer/Polymer Interfaces: A Joint Experimental and Theoretical Study. Phys. Rev. B: Condens. Matter Mater. Phys. 1999, 60, 5721-5727.

(46) Song, Y.; Clafton, S. N.; Pensack, R. D.; Kee, T. W.; Scholes, G. D. Vibrational Coherence Probes the Mechanism of Ultrafast Electron Transfer in Polymer-Fullerene Blends. Nat. Commun. 2014, 5, 4933.

(47) Dougherty, D. A. The Cation- $\pi$ Interaction. Acc. Chem. Res. 2013, 46 (4), 885-893. 
(48) Baughman, R. H.; Zakhidov, A. A.; de Heer, W. A. Carbon Nanotubes-the Route Toward Applications. Science 2002, 297, 787792.

(49) White, C. T.; Todorov, T. N. Carbon Nanotubes as Long Ballistic Conductors. Nature 1998, 393, 240-242.

(50) Kasahara, Y.; Tamura, R.; Tsukada, M. Structure and Electronic States of Capped Carbon Nanotubes by a Tight-binding Approach. Phys. Rev. B: Condens. Matter Mater. Phys. 2003, 67, 115419.

(51) Collins, P. G.; Zettl, A.; Bando, H.; Thess, A.; Smalley, R. E. Nanotube Nanodevice. Science 1997, 278, 100-102.

(52) Kataura, H.; Kumazawa, Y.; Maniwa, Y.; Umezu, I.; Suzuki, S.; Ohtsuka, Y.; Achiba, Y. Optical Properties of Single-Wall Carbon Nanotubes. Synth. Met. 1999, 103, 2555-2558.

(53) Cataldo, S.; Salice, P.; Menna, E.; Pignataro, B. Carbon Nanotubes and Organic Solar Cells. Energy Environ. Sci. 2012, 5, 5919-5940. 